\title{
ProwisT - Ein Instrument zur Einschätzung des professionellen Handlungswissens von Trainer/innen
}

\author{
Susanne Wißhak ${ }^{1,2}$ (D) Caroline Bonnes ${ }^{1}$ Sabine Hochholdinger ${ }^{1}$ \\ Angenommen: 2. April 2021 / Online publiziert: 27. April 2021 \\ (c) Der/die Autor(en) 2021
}

\section{Zusammenfassung}

Der vorliegende Beitrag in der Zeitschrift Gruppe. Interaktion. Organisation (GIO) beinhaltet die Vorstellung, theoretische Einordnung und inhaltliche Validierung eines Selbst- und Fremdbewertungsinstruments für Trainerwissen.

Trainer/innen sind Personen, die berufliche und betriebliche Fort- und Weiterbildungsangebote durchführen. Von ihren Kompetenzen und ihrem Wissen hängt die Qualität betrieblicher Weiterbildungen ab. Ausgehend von einer literaturbasierten Anforderungsermittlung wurde zunächst ein Fragebogen bezüglich des von Trainer/innen benötigten professionellen Handlungswissens entwickelt. Dieser Fragebogen lässt sich in einer modifizierten Form, dem Instrument zur Einschätzung des professionellen Handlungswissens von Trainer/innen (ProwisT), auch für die Selbst- oder Fremdeinschätzung des Wissens von Trainer/innen einsetzen. Das Instrument umfasst 41 Wissensfacetten, die sich auf 10 übergeordnete, theoretisch fundierte Wissensdimensionen verteilen. Dazu gehören neben dem Wissen über die Planung und Konzeption von Trainings Kenntnisse über Gruppenmanagement, die methodische Umsetzung von Trainings, Diagnostik, bildungswissenschaftliche Grundlagen, individuelle Unterschiede der Teilnehmenden, Fachwissen, fachdidaktisches Wissen, Organisationswissen sowie Kenntnisse über Beratung und Coaching.

Die 41 Wissensfacetten wurden zunächst von 200 Trainer/innen, 253 Teilnehmenden und 93 Personalverantwortlichen auf einer sechsstufigen Skala im Hinblick auf ihre Relevanz für die Trainertätigkeit beurteilt. Mit wenigen Ausnahmen wurde die Relevanz der 41 Facetten durch die Befragten bestätigt. Die Teilnehmenden scheinen bei der Bewertung der Facetten teilweise andere Schwerpunkte zu setzen als Trainer/innen und Personalverantwortliche.

Das modifizierte Instrument ProwisT kann von Trainer/innen zum Abgleich des eigenen professionellen Wissens und damit für eine individualisierte Kompetenzentwicklung genutzt werden. Außerdem kann es als Standard für Trainerausbildungen, Trainingsanbieter und Personalabteilungen dienen.

Schlüsselwörter Beruflich-betriebliche Weiterbildung · Training · Instrument ProwisT · Professionelles Handlungswissen von Trainer/innen $\cdot$ Kompetenz $\cdot$ Fragebogenentwicklung

\section{ProwisT_An Instrument for Assessing the Professional Knowledge and Skills of Trainers}

\begin{abstract}
The present article in the journal Gruppe. Interaktion. Organisation (GIO) includes the introduction, theoretical classification and content validation of a self- and external assessment tool for trainer knowledge.
\end{abstract}

Susanne Wißhak

Susanne.Wisshak@uni-konstanz.de

1 Lehrstuhl für Betriebspädagogik, Universität Konstanz, Konstanz, Deutschland

2 Lehrstuhl für Betriebspädagogik, Fach 125, Universität Konstanz, 78457 Konstanz, Deutschland 
Trainers are individuals who provide work-related continuing education and training. The quality of training depends on their competence and knowledge. Proceeding from a literature-based determination of requirements, a questionnaire was first developed regarding the professional knowledge and skills required by trainers. This questionnaire can also be used in a modified form, the instrument for the assessment of the professional knowledge and skills of trainers (ProwisT), for the self-evaluation or external evaluation of trainers' knowledge. The instrument comprises 41 knowledge facets, which are distributed across 10 superordinate, theoretically sound knowledge dimensions. In addition to knowledge about the planning and designing of training, these include knowledge about group management, the methodical implementation of training, diagnostics, educational science basics, individual differences of the participants, content knowledge, pedagogical content knowledge, organizational knowledge as well as knowledge about consulting and coaching.

The 41 knowledge facets were initially assessed by 200 trainers, 253 participants and 93 HR managers on a six-point scale with regard to their relevance for trainers. With few exceptions, the relevance of the 41 facets was confirmed by the respondents. The participants seem to have partly different priorities in the evaluation of the facets than trainers and HR managers.

The modified instrument ProwisT can be used by trainers to evaluate their own professional knowledge and thus for individualized competence development. It can also serve as a standard for train-the-trainer programs, training providers and HR departments.

Keywords Continuing professional education · Training · Survey instrument ProwisT · Professional knowledge and skills of trainers · Competence $\cdot$ Questionnaire development

\section{Professionelles Handlungswissen von Trainer/innen}

Trainer/innen sind Personen, die berufliche und betriebliche Weiterbildungsveranstaltungen planen und durchführen (Fuchs 2011). Der Begriff Trainer/innen wird im vorliegenden Beitrag im Sinne einer breiten Definition verwendet, d.h. unabhängig von Kontext (z.B. Unternehmen, Bildungsanbieter, freie Bildungsträger), Beschäftigungsverhältnis (z.B. freiberuflich, festangestellt), Weiterbildungsformaten und Zielgruppen.

Verschiedene Befunde legen nahe, dass das professionelle Handlungswissen von Trainer/innen die Qualität der Weiterbildungsmaßnahmen beeinflussen kann (Hunter 1986; Voss et al. 2014). Mit professionellem Handlungswissen sind die für berufliches Handeln nötigen kognitiven Fähigkeiten und Fertigkeiten gemeint. Es stellt einen Teilbereich der professionellen Handlungskompetenz dar, die neben dem Wissen auch motivationale, volitionale und soziale Handlungsvoraussetzungen umfasst und als Voraussetzung für die Lehrperformanz angesehen wird (Baumert und Kunter 2006; Blömeke et al. 2014; Kunter et al. 2013; Weinert 2002). Es wird also davon ausgegangen, dass professionelles Handlungswissen, gemeinsam mit kognitiven Fähigkeiten und affektiv-motivationalen Dispositionen, eine zentrale Rolle für kompetentes Handeln in komplexen Handlungsdomänen, wie Trainertätigkeiten, spielt (Blömeke et al. 2015).

Trotz ihrer Relevanz für gelingende Personalentwicklung ist bislang unzureichend erforscht, welches professionelle Handlungswissen Trainer/innen benötigen, und wie dieses Wissen erfasst werden kann. Daher wird in diesem
Beitrag ein entsprechendes Instrument vorgestellt. Im Rahmen der Studie Trainerwissen wurde zunächst die Relevanz verschiedener Wissensfacetten durch Trainer/innen, Teilnehmende und Personalverantwortliche beurteilt (Wißhak und Hochholdinger 2018). Der vorliegende Beitrag enthält eine modifizierte Version des Fragebogens, das Instrument zur Einschätzung des professionellen Handlungswissens von Trainer/innen (ProwisT), das für die Selbstoder Fremdbeurteilung von Trainerwissen verwendet werden kann. Hierfür wurden die Items aus dem ursprünglichen Fragebogen so umformuliert, dass sie sich nicht mehr auf das von Trainer/innen generell benötigte Handlungswissen beziehen, sondern darauf, ob die Person, die den Fragebogen ausfüllt, das jeweilige Wissen besitzt. Das Instrument kann auf individueller und auf organisationaler Ebene unterstützend bei der Kompetenzentwicklung eingesetzt werden und damit einen Beitrag zur Professionalisierung von Trainer/innen leisten, z. B. bei der individuellen Reflexion und Weiterentwicklung als auch im Rahmen eines organisationalen Kompetenzmanagements. Darüber hinaus kann es ergänzend bei der Auswahl und Einstellung von Trainer/ innen verwendet werden.

Für die konzeptionelle Einordnung des Konstrukts Trainerwissen wird im folgenden Abschnitt zunächst das übergreifende Kompetenzmodell des Projekts GRETA des Deutschen Instituts für Erwachsenenbildung (DIE) herangezogen, das für Lehrende in allen Weiterbildungsbereichen entwickelt wurde (Lencer und Strauch 2016). Es werden außerdem Befunde aus der Trainingsforschung zusammengefasst, die einen Überblick über die Forschungslage geben sollen und gleichzeitig die Grundlage für den Fragebogen ProwisT darstellen. In Abschnitt drei wird das Instru- 
ment vorgestellt. Außerdem wird auf die Beurteilung der Wissensfacetten durch Trainer/innen, Teilnehmende und Personalverantwortliche eingegangen. In Abschnitt vier werden die Limitationen des Instruments erörtert, bevor in Abschnitt fünf der praktische Nutzen diskutiert wird.

\section{Modelle und Befunde der Trainingsforschung}

Bisher existiert kein anerkanntes, wissenschaftlich fundiertes Modell, das sich eigens mit Trainerkompetenzen beschäftigt (Bonnes et al. 2019). Modelle der Trainingsforschung fokussieren zumeist den Transfer der im Training erworbenen Fähigkeiten auf den Arbeitsplatz und die damit verbundenen Determinanten des Trainingstransfers. Das etablierteste Modell von Baldwin und Ford (1988) wie auch neuere Modelle (z.B. Blume et al. 2019) lassen Trainer/ innen als Einflussfaktor meist unberücksichtigt. Eine Ausnahme bildet das Transfermodell von Burke und Hutchins (2008). Trainer/innen werden hier zwar mitberücksichtigt, es wird jedoch nicht vertieft auf die notwendigen Kompetenzen eingegangen. Daher wird hier zunächst das GRETAKompetenzmodell vorgestellt, welches generell für Lehrende in der Weiterbildung entwickelt wurde (Lencer und Strauch 2016). Anschließend werden ergänzend einige der Befunde aus der internationalen Trainingsforschung zusammengefasst, auf welchen das Instrument ProwistT basiert.

\subsection{Das Kompetenzmodell des GRETA Projekts}

Im Rahmen des Projekts GRETA I wurde ein Modell entwickelt, welches Kompetenzen abbilden soll, die für eine professionelle Lehrtätigkeit in der Erwachsenen- und Weiterbildung benötigt werden (siehe Abb. 1; Lencer und Strauch 2016). Auf dieser Basis können non-formal und informell erworbene Kompetenzen von Lehrenden anerkannt sowie Qualifizierungsbedarfe und -optionen ausgelotet werden. Zurzeit werden die Anerkennungsinstrumente in der zweiten Projektphase (GRETA II) erprobt (Bosche et al. 2019).
Abb. 1 Kompetenzmodell des GRETA Projekts (Lencer und Strauch 2016). Übernommen mit freundlicher Erlaubnis des Deutschen Instituts für Erwachsenenbildung, Leibniz-Zentrum für Lebenslanges Lernen e. V

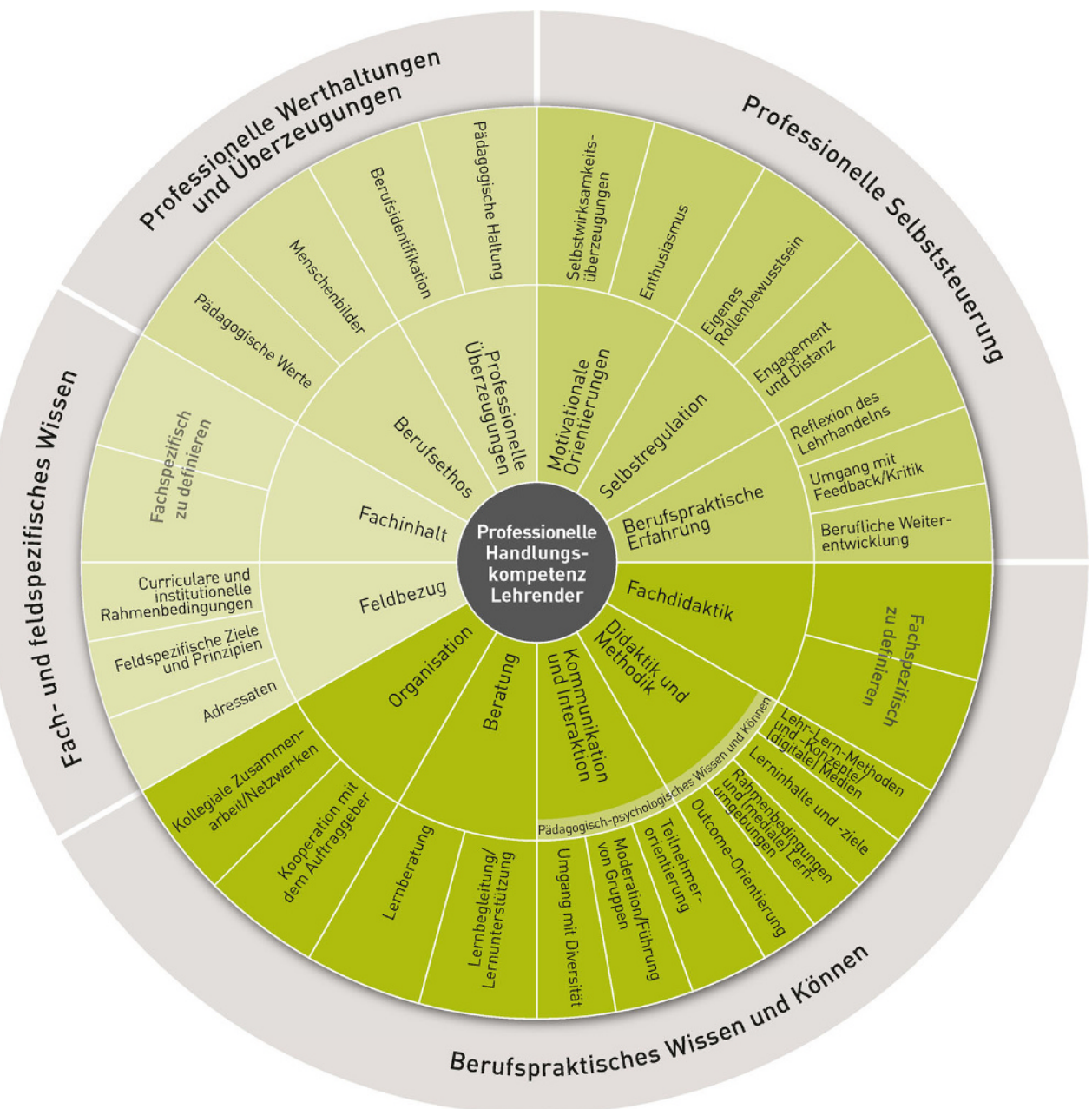


Das Modell unterscheidet vier Kompetenzaspekte (Lencer und Strauch 2016):

- Berufspraktisches Wissen und Können

- Fach- und feldspezifisches Wissen

- Professionelle Selbststeuerung sowie

- Professionelle Werthaltungen und Überzeugungen.

Diese Kompetenzaspekte sind in Kompetenzbereiche und diese wiederum in Kompetenzfacetten untergliedert. Für den vorliegenden Beitrag sind die ersten beiden o.g. Kompetenzaspekte relevant, da sie sich auf das Handlungswissen der Lehrenden beziehen. Zum berufspraktischen Wissen und Können gehören die Bereiche Organisation, Beratung, Kommunikation und Interaktion, Didaktik und Methodik sowie Fachdidaktik. Unter den Kompetenzbereich des fach- und feldspezifischen Wissens fallen die Fachinhalte sowie der Feldbezug.

Obwohl das Modell eine gute Einordnung des hier vorgestellten Instruments in die Kompetenzen von Lehrenden in der Erwachsenen- und Weiterbildung ermöglicht, gibt es keinen Aufschluss darüber, welches Wissen speziell von Trainer/innen in der beruflichen und betrieblichen Weiterbildung benötigt wird. Während sich das GRETAModell an der empirischen Unterrichtsforschung (Baumert und Kunter 2006) und der Erwachsenenbildungsforschung orientiert, wurde daher im Hinblick auf das Instrument ProwisT bei der literaturbasierten Anforderungsermittlung für Trainer/innen die internationale Trainingsforschung herangezogen.

\subsection{Befunde der internationalen Trainingsforschung}

Die internationale Trainingsliteratur ist für die Ermittlung des erforderlichen Trainerwissens in zweierlei Hinsicht relevant. Zum einen liefert sie Informationen zu Determinanten des Trainingserfolgs, und zum anderen existieren einige wenige Untersuchungen $\mathrm{zu}$ erfolgsrelevanten Trainermerkmalen. Im Folgenden werden beide Forschungsbereiche kurz vorgestellt.

Zunächst existiert eine Vielzahl an Studien, die sich mit Erfolgsfaktoren von Trainings beschäftigen. Als Indikator für Trainingserfolg wird hier neben dem Wissens- und Fähigkeitserwerb der Teilnehmenden meist der Transfer des Gelernten auf den Arbeitsplatz untersucht. Dieser ist zentral für berufliche und betriebliche Trainings.

Die Fülle an Originalstudien lässt sich anhand einiger Meta-Analysen und Literaturreviews überblicken (z. B. Bell et al. 2017; Ford et al. 2018). Sie behandeln überwiegend Determinanten, die die Teilnehmenden, das Trainingsdesign, oder Merkmale des Arbeits- bzw. Anwendungskontexts betreffen (Baldwin und Ford 1988). Das von Trainer/ innen benötigte Wissen wird hierbei nicht explizit unter- sucht. Jedoch lässt sich anhand dieser Studien das benötigte professionelle Handlungswissen aus den gefundenen transferrelevanten Faktoren ableiten. Somit ergeben sich folgende Anforderungen an Trainer/innen in Bezug auf ihr Wissen zu den Faktoren Teilnehmermerkmale, Trainingsdesign und Arbeitsumfeld.

Bezüglich der Teilnehmenden sollten Trainer/innen Wissen über mögliche individuelle Unterschiede und Voraussetzungen besitzen. So können bspw. Intelligenz oder verschiedene Persönlichkeitsmerkmale mit dem Transfererfolg zusammenhängen (Blume et al. 2010; Colquitt et al. 2000). Bezüglich der Teilnehmermerkmale sind vor allem die Motivation und die Selbstwirksamkeit diejenigen Faktoren, welche die Trainer/innen beeinflussen können. Sie sollten vor, während und nach dem Training gestärkt werden (Colquitt et al. 2000; Gegenfurtner 2011; Gegenfurtner et al. 2013; Hughes et al. 2020).

Bezüglich des Trainingsdesigns sollten Trainer/innen wissen, wie sie Trainings wirksam konzipieren und gestalten können, indem sie bestimmte Methoden einsetzen (Arthur et al. 2003; Keith und Frese 2008; Taylor et al. 2005), das Training auf die Bedarfe der Lernenden und die Lernziele zuschneiden, die Teilnehmenden in Entscheidungsprozesse einbeziehen (Gegenfurtner et al. 2016), die Relevanz der Trainingsziele kommunizieren (Colquitt et al. 2000), den Teilnehmenden ausreichend Gelegenheit geben, das erwünschte Verhalten zu üben (Driskell et al. 1992), ihnen regelmäßig Feedback geben und ihnen positive und negative Lernmodelle anbieten (Taylor et al. 2005). Hierfür müssen Trainer/innen wissen, wie sie in allen Phasen eines Trainings den Lernerfolg und den Transfer fördern können.

Bezüglich des Arbeitsumfelds sollten Trainer/innen den organisationalen Kontext in allen Trainingsphasen mitbedenken, also den Trainingsbedarf erheben, die Führungskräfte und Kolleg/innen mit einbinden (Colquitt et al. 2000; Hughes et al. 2020; Reinhold et al. 2018) und den Teilnehmenden vermitteln, dass von ihnen erwartet wird, dass sie das Gelernte am Arbeitsplatz anwenden (Huang et al. 2015).

Neben diesen Wirkungsstudien existieren einige Untersuchungen, die sich speziell mit Trainermerkmalen beschäftigen. Meistens wurden dabei Praktiker/innen dazu befragt, welche Attribute erfolgreiche Trainer/innen ausmachen. Im Folgenden werden die wichtigsten dieser Studien zu Trainer/innen kurz vorgestellt, einen Überblick über die Forschungslage liefern auch Bonnes et al. (2019).

Gauld und Miller (2004) konstruierten auf Basis einer Delphi-Studie einen Online-Fragebogen und befragten 303 Trainer/innen zu ihrer Berufserfahrung, ihren Qualifikationen und der Relevanz mehrerer Kompetenzen. Die 45 Trainer/innen, die mindestens zehn Jahre Berufserfahrung sowie eine formale Trainerqualifikation besaßen, schätzten die folgenden Kompetenzen als besonders wichtig ein: Ziele set- 
zen, Reflexion, Evaluation, positive Verstärkung, Lernaktivitäten in Gruppen ermöglichen, faire Leistungsmessung, aktives Zuhören, Anforderungsanalyse, Beratung und das Einbeziehen der Teilnehmenden durch Fragen.

Burke und Hutchins (2008) erhoben bei 139 Praktiker/ innen in einem offenen, schriftlichen Format Best Practice-Strategien zur Förderung von Trainingstransfer. Neben anderen Aspekten wurden auch drei Kategorien von Trainermerkmalen genannt: Fachwissen, Berufserfahrung und Wissen über Lehrprinzipien, wobei unter letzteres bspw. Flexibilität und Teilnehmerorientierung fallen.

In einer weiteren qualitativen Untersuchung ermittelten Nisar und Scott (2011) mithilfe halbstrukturierter Interviews und Fokusgruppen bei zehn Trainer/innen und 32 Teilnehmenden eines chirurgischen Trainingsprogramms als wichtig erachtete Trainerattribute. Diese wurden sechs Themen zugeordnet: Führung und Organisation, Verpflichtung/Engagement bezüglich des Trainings, die Teilnehmenden als Individuen behandeln, moderne Methoden und Lehrpläne verwenden, effektiv kommunizieren und medizinische Exzellenz. Am häufigsten wurden Attribute der ersten Kategorie, Führung und Organisation genannt. Dazu gehörte u.a., dass die Lehrperson als Vorbild fungiert, die Teilnehmenden aber auch Fälle selbst bearbeiten lässt. Die zweithäufigste Kategorie, Kommunikation, beinhaltete u. a., dass die Lehrperson Schwierigkeiten mit den Lernenden bespricht, enthusiastisch ist und Feedback gibt.

Matsuo (2014) entwickelte in einem mehrschrittigen Verfahren vier Dimensionen instruktionaler Fähigkeiten von Inhouse-Trainer/innen: Reflexion über Ergebnisse fördern, Fortschritt überwachen, positives Feedback geben und anspruchsvolle Ziele stecken.

In einer weiteren Studie sammelten Hodge und Harvey (2015), basierend auf einem Literaturreview und Expertenbefragungen, 14 Expertise-Bereiche von Personalentwickler/innen und ließen diese von 589 australischen Personalentwickler/innen bezüglich ihrer Relevanz beurteilen. Die höchste Zustimmung erfuhren die Bereiche Strategie, Bedarfsanalyse, Design, Lernendenmerkmale und Unterstützung.

\section{Beschreibung des Instruments ProwisT}

Für die Entwicklung des Fragebogens wurde zunächst im Rahmen der Studie Trainerwissen ein Literaturreview zu relevanten Trainermerkmalen und Prinzipien effektiver Trainingsgestaltung durchgeführt (Wißhak und Hochholdinger 2018). Einige der verwendeten Studien wurden in Abschn. 2 vorgestellt. Die im Rahmen des Literaturreviews gefundenen Einzelbefunde wurden zu 41 Wissensfacetten zusammengefasst (Mayring 2010) und zu zehn Dimensionen geordnet (Tab. 1). Bei der Entwicklung der Dimensionen wur- den auch gängige Modelle zu Lehrerkompetenzen und -wissen berücksichtigt (z. B. Baumert und Kunter 2006; König und Blömeke 2009; Kunina-Habenicht et al. 2012). Für jede Wissensfacette wurde ein Item formuliert, wobei darauf geachtet wurde, dass eine Sprache verwendet wird, die Akteuren aus der betrieblichen und beruflichen Weiterbildung geläufig ist. Die meisten Items wurden zusätzlich mit kurzen Erklärungen oder Beispielen versehen. Da die meisten Trainer/innen keine akademische pädagogische Vorbildung besitzen (Wißhak et al. 2020), ist es möglich, dass ihnen Begriffe wie didaktische Prinzipien u. U. nicht vertraut sind, obwohl sie solche durchaus kennen und nutzen.

Die Items werden auf einer sechsstufigen Likert-Skala mit den Ausprägungen trifft gar nicht zu bis trifft völlig $z u$ bewertet. Während in der Version des Fragebogens, die für die Trainerwissen-Studie verwendet wurde, nach der Relevanz der Wissensfacetten für den Trainerberuf gefragt wurde, wird in ProwisT danach gefragt, ob eine Person das jeweilige Handlungswissen besitzt.

Der Fragebogen kann online oder als Paper-Pencil-Fragebogen eingesetzt werden, wobei die Bearbeitung etwa zehn Minuten erfordert.

\subsection{Wissensfacetten und Wissensdimensionen}

Unter die Dimension A Didaktische Planung und Konzeption von Trainings fallen die Definition der Lernziele sowie die Trainingskonzeption und didaktische Modelle und Prinzipien. Zu der Dimension B Wissen über Gruppenmanagement gehören bspw. der Umgang mit Konflikten, die Leitung von Gruppen, Kommunikation und Beziehungsgestaltung. Die Dimension C umfasst die methodische Umsetzung eines Trainings. Dazu gehören u.a. die Kenntnis verschiedener Methoden und deren Variation, die Verwendung von Medien und Lernmaterialien, die kognitive Aktivierung und Motivation der Teilnehmenden, Feedback sowie die Konsolidierung des Gelernten. Unter die Dimension D Wissen über Diagnostik fallen die Bedarfsanalyse, Diagnostik, Forschungswissen, Überprüfung der Lernerfolge und die Evaluation von Trainings. $\mathrm{Zu}$ der Dimension E Bildungswissenschaftliches Grundlagenwissen zählen theoretische Grundlagen von Bildung und Erziehung sowie Wissen über das deutsche Bildungssystem, Wissen über Lernprozesse und Kenntnis der empirischen Trainingsforschung. Die Dimension $F$ umfasst die beiden Wissensfacetten Teilnehmerorientierung und Umgang mit Heterogenität. Zu der Dimension G Fachwissen gehört neben dem Wissen über die Trainingsinhalte auch die berufspraktische Erfahrung der Trainer/innen. Unter die Dimension H Fachdidaktisches Wissen fallen Erklärungswissen, Wissen über die Vorstellung der Teilnehmenden zum Trainingsthema sowie fachspezifische Trainingsmethoden. Die Dimension I, Organisationswissen, umfasst neben unternehmerischem Wissen 
Tab. 1 Instrument zur Einschätzung des professionellen Handlungswissens von Trainer/innen (ProwisT)

Dimension A: Wissen über die didaktische Planung und Konzeption von Trainings M (SD)

Ich weiß, wie ich anspruchsvolle Lernziele festlege.

$4,92(1,09)$

Lernziele werden vor dem Training festgelegt und beschreiben Wissen, Fertigkeiten oder Verhalten, die die Teilneh-

menden nach dem Training zeigen sollen

Ich weiß, wie ich Trainings konkret konzipiere.

D.h., wie ich basierend auf meiner Bedarfsanalyse und meinen Lernzielen die Inhalte aufbereite, das Format des Trai-

nings auswähle, den Ablauf und die Lernformen plane und das Material erstelle

Ich kenne didaktische Modelle.

Diese bilden Lehr-Lernszenarien ab. Dazu gehören z. B. die Didaktik nach Klafki, konstruktivistische Ansätze oder

Verhaltensmodellierung

Ich kenne didaktische Prinzipien der Trainingsplanung.

Damit sind allgemeine didaktische Handlungsempfehlungen gemeint, wie z. B. kognitive Aktivierung, Praxisbezug,

Anschaulichkeit

Dimension B: Wissen über Gruppenmanagement

Ich weiß, wie ich am besten mit Konflikten umgehe, die im Training entstehen können

indem ich Konfliktlösungsstrategien beherrsche und zur Störungsprävention Regeln und Rituale etabliere

Ich weiß, wie ich die Trainingszeit möglichst effektiv nutze, so dass ein hoher Anteil aktiver Lernzeit ermöglicht wird.

Z. B. indem ich ein gutes Zeitmanagement beherrsche

Ich weiß, wie ich die Lernumgebung so gestalte, das die Teilnehmenden sie als konstruktiv und unterstützend erleben.

Z. B. indem ich ein lernförderliches Klima herstelle, anregende Lernumgebungen biete, für Fragen stets verfügbar bin, geduldig bleibe und mit Fehlern konstruktiv umgehe

Ich weiß, wie ich flexibel auf unvorhergesehene Situationen reagieren kann, ohne dass der Gesamtablauf beeinträchtigt wird

Ich weiß, wie ich Gruppenprozesse erkennen und steuern kann

Ich weiß, wie ich im Training adäquat kommuniziere.

Z.B. indem ich Gesprächs- und Kommunikationstechniken beherrsche und angemessen auftrete

Ich weiß, wie ich eine möglichst positive und konstruktive Beziehung zu den Teilnehmenden aufbaue und aufrecht erhalte

Dimension C: Wissen über die methodische Umsetzung eines Trainings

Ich weiß, wie ich den Teilnehmenden Lernziele transparent machen kann

indem ich diese klar kommuniziere und Wege aufzeige, wie diese erreicht werden können

Ich weiß, wie ich die Trainingssequenzen sinnvoll aufeinander aufbaue, damit ein roter Faden entsteht

$5,26(1,02)$

$4,28(1,37)$

$5,24(0,98)$

$5,15(1,09)$

$5,20(0,99)$

$5,37(0,91)$

$4,98(1,13)$

$5,48(0,87)$

$5,40(0,96)$

$5,36(0,89)$

$5,49(0,89)$

$4,95(1,16)$

Ich kenne verschiedene Lehr-Lernmethoden und weiß, wie ich sie zielorientiert einsetzen und variieren kann

$5,16(0,98)$

Ich weiß, wie ich Medien und Lernmaterialien gezielt und sinnvoll einsetze.

Z. B. wann ich eine PowerPoint-Präsentation mache, mit dem Flipchart oder einer Metaplanwand arbeite, wie ich ein

Handout oder Skript erstelle, etc

Ich weiß, wie ich die Teilnehmenden kognitiv (= geistig) aktivieren kann.

Z.B. durch herausfordernde Aufgaben, die eigenständig oder kooperativ gelöst werden müssen, durch praktische Übungen oder komplexe Situationen

Ich weiß, wie ich die Teilnehmenden motivieren kann.

Z.B. wie ich Anreize setze, Freude am Lernen vermittle und Praxisbezüge aufzeige

Ich weiß, wie ich optimale Voraussetzungen dafür schaffe, dass das Gelernte in die Praxis übertragen wird

indem ich über die Prozesse, Förderung und Hürden von Lerntransfer Bescheid weiß

Ich weiß, wie ich Feedback an die Teilnehmenden gebe.

Z.B. wann ich welche Feedbackform wähle und wie ich dieses klar, aussagekräftig und lernförderlich formulieren kann

Ich weiß, wie ich sicherstelle, dass das Gelernte behalten wird.

Z. B. durch wiederholtes Üben, Merkhilfen, Feedback

Dimension D: Wissen über Diagnostik

Ich weiß, wie ich vor dem Training eine Bedarfsanalyse durchführe.

Eine Bedarfsanalyse ermittelt auf Basis systematischer Erhebungen, welche Inhalte für welche Zielgruppe in welcher

Organisation vermittelt werden sollen.

Ich weiß, mit welchen Testverfahren ich die aktuellen Kompetenzen meiner Teilnehmenden systematisch diagnostizieren und die Ergebnisse auswerten kann.

Z.B. über systematische Lernstandserhebungen, Wissenstests, psychologische Fähigkeitstests

Ich beherrsche verschiedene Forschungsmethoden.

Z.B. um meine Trainings systematisch zu evaluieren und ihren Effekt statistisch auszuwerten

$5,19(0,93)$

$5,32(0,96)$

$5,08(1,05)$

$4,91(1,31)$

$3,49(1,38)$

$3,35(1,36)$ 
Tab. 1 (Fortsetzung)

Ich weiß, mit welchen Methoden ich die Lernerfolge der Teilnehmenden überprüfen kann.

$4,37(1,25)$

Z.B. mit Wissenstests oder Verhaltensbeurteilung

Ich weiß, wie ich mein Training evaluieren kann.

Damit ist nicht nur das Abfragen der Zufriedenheit der Teilnehmenden gemeint, sondern auch die Messung der erworbenen Kenntnisse und des Transfers.

Dimension E: Bildungswissenschaftliches Grundlagenwissen

Ich weiß über die theoretischen Grundlagen von Erziehung und Bildung Bescheid.

Z. B. über Grundbegriffe, Theorien und Geschichte der Erziehungs- und Bildungswissenschaft

Ich kenne mich mit dem deutschen Bildungssystem aus

$4,87(1,17)$

Z. B. über Gedächtnismodelle, Lerntheorien, Wissenserwerb, Lern- und Leistungsmotivation

Ich weiß über aktuelle empirische Trainingsforschung Bescheid

indem ich z. B. regelmäßig Fachartikel lese

Dimension F: Wissen über individuelle Unterschiede der Teilnehmenden

Ich weiß, wie ich mein Training auf die Teilnehmenden (als Gruppe und als Individuen) zuschneiden kann indem ich die fachlichen und persönlichen Voraussetzungen sowie Erwartungen berücksichtige

Ich weiß, wie ich mit Heterogenität innerhalb des Trainings umgehen soll.

Z.B. Unterschiede bzgl. Leistungsniveau, Lernbiografie, Alter, Herkunft

Dimension G: Fachwissen

Ich weiß über die in meinem Training zu vermittelnden Inhalte Bescheid.

Z.B. kenne ich mich als Software-Trainer*in sehr gut mit dem Computerprogramm aus. Als Führungstrainer*in bin ich vertraut mit Führungstheorien etc

Ich besitze eigene praktische Erfahrung mit dem Trainingsthema.

Z. B. habe ich als Vertriebstrainer*in bereits selbst im Vertrieb gearbeitet

Dimension H: Fachdidaktisches Wissen

Ich weiß, wie ich mein Trainingsthema am besten erklären kann.

Z.B. durch verschiedene anschauliche Beispiele oder verschiedene, alternative Erklärungsansätze

Ich weiß, welche Vorstellungen die Teilnehmenden zum Trainingsthema mitbringen.

Z. B. bekanntes Vorwissen, aber auch typische Fehler oder verbreitete Mythen

Ich weiß, welche Methoden speziell für mein Trainingsthema besonders geeignet sind.

Z. B. Behavior Modeling Training bei Kommunikationstrainings, Simulationen für technische Systeme, themenspezifi-

sche Übungen, etc

Dimension I: Organisationswissen

Ich besitze unternehmerisches Wissen.

Z. B. über Marketing, Akquise, Buchhaltung

Ich weiß, wie Organisationsentwicklungs- und Changemanagement-Prozesse funktionieren.

Z. B. Ablauf und Phasen, Methoden der Organisationsdiagnose, Organisationstheorien

Ich besitze Wissen über Personalmanagement und Personalentwicklung in Organisationen.

Z. B. Verfahren der Personalauswahl, Anreizsysteme, Vergütung, Leistungsbeurteilung, Bildungsmanagement, Karriereplanung, strategische Personalentwicklung

Dimension J: Wissen über Beratung und Coaching

Ich weiß, wie ich Beratungsgespräche führe.

Z.B. wie ich sie strukturiere

Ich weiß, wie ich ein Coaching durchführe.

Unter Coaching verstehen wir eine individuelle Unterstützung des/r Coachee durch eine/n Coach, welche auf der Prozessebene stattfindet. Der/die Coach erteilt dabei keine Ratschläge, sondern begleitet den/die Coachee bei eigenverantwortlichen Entscheidungen

Durchschnittliche Relevanzeinschätzungen aller Befragten $(546>N>511)$. Skala: $1=$ trifft gar nicht $z u, 6=$ trifft völlig zu (siehe auch Wißhak und Hochholdinger 2018, S. 226). Für die Verwendung des Fragebogens empfehlen wir, die 41 Items in einer zufälligen Reihenfolge zu bearbeiten, ohne die Dimensionen zu benennen. 
das Wissen über Organisationentwicklung und Change-Management sowie das Wissen über Personalmanagement und Personalentwicklung. Die letzte Dimension $J$ schließlich beinhaltet Wissen über Beratung und Coaching.

Die zehn Wissensdimensionen des Instruments finden sich größtenteils auch in den wissensbezogenen Kompetenzaspekten des GRETA-Modells (Lencer und Strauch 2016) wieder, allerdings ist ProwisT durch die konkreten Wissensfacetten etwas differenzierter; so haben wir bspw. versucht, fachdidaktische Items zu bilden, obwohl die fachlichen Inhalte sehr divers sein können. Darüber hinaus fragt ProwisT vor allem nach professionellem Handlungswissen, das speziell für die berufsbezogene Weiterbildung und die spezifischen Anforderungen an Trainer/innen wichtig ist, wie z. B. Wissen über Trainingstransfer oder Entwicklungsprozesse in Organisationen.

\subsection{Bewertung der Wissensfacetten durch verschiedene Stakeholder}

Um zu überprüfen, ob die aus der Literatur abgeleiteten Wissensfacetten auch aus der Sicht zentraler Trainingsakteure Relevanz für die Trainertätigkeit besitzen, wurde zunächst die Studie Trainerwissen durchgeführt (Wißhak und Hochholdinger 2018). Hierfür bewerteten 200 Trainer/ innen, 253 Teilnehmende und 93 Personalverantwortliche die 41 Wissensfacetten dahingehend, ob Trainer/innen das Wissen für ihre Tätigkeit benötigen. Die Gelegenheitsstichprobe wurde über verschiedene Kanäle, wie Weiterbildungsanbieter und berufliche Online-Netzwerke rekrutiert, an die der Fragebogenlink verschickt wurde.

Die Bewertungen der Items durch die 546 Befragten auf den sechsstufigen Likert-Skalen wurden mit der Statistiksoftware SPSS (IBM Corp 2020) zunächst deskriptiv aus- gewertet bevor zur Ermittlung von Gruppenunterschieden eine multivariate Varianzanalyse (MANOVA) und eine Diskriminanzanalyse berechnet wurden.

Während die Trainer/innen ( $M=48$ Jahre, 102 weiblich) und die Personalverantwortlichen ( $M=49$ Jahre, 49 weiblich) im Mittel ähnlich alt waren, waren die Teilnehmenden durchschnittlich etwa zehn Jahre jünger (38 Jahre, 125 weiblich). Die meisten Trainer/innen gaben an, für Unternehmen zu arbeiten (148). Für Industrie- und Handelskammern arbeiteten 46 Trainer/innen und 49 im Umfeld von Hochschulen (Mehrfachnennungen möglich). Viele Trainer/innen (66) gaben aber auch sonstige Weiterbildungsbereiche an, wie diverse Weiterbildungsanbieter (Volkshochschulen, Trainingsanbieter), Arztpraxen und Kliniken, öffentliche Verwaltung sowie offene Trainings für Privatpersonen.

Die deskriptiven Werte für die 41 Items sind in Tab. 1 abgebildet. In Tab. 2 finden sich die Mittelwerte und Standardabweichungen der zehn Dimensionen. Wie erwartet, erfuhren die meisten Items eine hohe Zustimmung durch die Befragten $(M=4,78, S D=0,64)$. Auf der Ebene der einzelnen Items erfuhr das Item Wissen über die Trainingsinhalte die höchste Zustimmung $(M=5,52, S D=0,92)$. Es folgen die Strukturierung von Trainings $(M=5,49, S D=0,89)$, Kommunikation $(M=5,48, S D=0,87)$ und themenspezifische Methoden $(M=5,42, S D=0,92)$. Die niedrigste $\mathrm{Be}-$ wertung erhielten die theoretischen Grundlagen von Bildung und Erziehung $(M=3,35, S D=1,39)$, Forschungswissen $(\mathrm{M}=3,35, \mathrm{SD}=1,36)$ und Wissen über das Bildungssystem $(M=2,97, S D=1,48)$. Von den letztgenannten Ausnahmen abgesehen zeigte sich damit insgesamt eine hohe Zustimmungstendenz.

Für die jeweiligen Dimensionen wurde Cronbachs Alpha als Maß für die interne Konsistenz der zugehörigen

Tab. 2 Bewertung der Wissensdimensionen durch Trainer/innen, Teilnehmende und Personalverantwortliche

\begin{tabular}{|c|c|c|c|c|c|c|}
\hline \multirow{2}{*}{\multicolumn{2}{|c|}{ Wissensdimension }} & \multirow{3}{*}{$\frac{\propto}{0,77}$} & \multirow{3}{*}{$\begin{array}{l}\text { Gesamt } \\
M(S D) \\
4,95(0,86)\end{array}$} & \multirow{3}{*}{$\begin{array}{l}T R \\
5,01(0,86)\end{array}$} & \multirow{3}{*}{$\begin{array}{l}N \\
4,80(0,87)\end{array}$} & \multirow{3}{*}{$\begin{array}{l}P V \\
5,20(0,75)\end{array}$} \\
\hline & & & & & & \\
\hline A & $\begin{array}{l}\text { Wissen über die didaktische Planung und } \\
\text { Konzeption von Trainings }\end{array}$ & & & & & \\
\hline B & Wissen über Gruppenmanagement & 0,88 & $5,26(0,76)$ & $5,47(0,67)$ & $4,98(0,82)$ & $5,56(0,43)$ \\
\hline $\mathrm{C}$ & $\begin{array}{l}\text { Wissen über die methodische Umsetzung } \\
\text { eines Trainings }\end{array}$ & 0,87 & $5,24(0,68)$ & $5,35(0,66)$ & $5,08(0,73)$ & $5,45(0,41)$ \\
\hline $\mathrm{D}$ & Wissen über Diagnostik & 0,76 & $4,08(0,94)$ & $4,02(0,95)$ & $4,08(0,96)$ & $4,19(0,90)$ \\
\hline $\mathrm{E}$ & Bildungswissenschaftliches Grundlagenwissen & 0,72 & $3,70(1,02)$ & $3,60(1,01)$ & $3,77(1,06)$ & $3,76(0,95)$ \\
\hline $\mathrm{F}$ & $\begin{array}{l}\text { Wissen über individuelle Unterschiede der } \\
\text { Teilnehmenden }\end{array}$ & 0,66 & $5,07(0,94)$ & $5,32(0,86)$ & $4,79(0,99)$ & $5,33(0,73)$ \\
\hline G & Fachwissen & 0,58 & $5,27(0,91)$ & $5,17(0,97)$ & $5,40(0,87)$ & $5,17(0,80)$ \\
\hline $\mathrm{H}$ & Fachdidaktisches Wissen & 0,63 & $5,20(0,76)$ & $5,31(0,70)$ & $5,05(0,83)$ & $5,36(0,60)$ \\
\hline I & Organisationswissen & 0,75 & $3,94(1,17)$ & $4,11(1,21)$ & $3,80(1,18)$ & $3,95(1,02)$ \\
\hline $\mathrm{J}$ & Wissen über Beratung und Coaching & 0,59 & $4,42(1,21)$ & $4,30(1,28)$ & $4,55(1,18)$ & $4,34(1,13)$ \\
\hline
\end{tabular}

Die 41 Wissensfacetten wurden den theoriebasierten Wissensdimensionen A-J zugeordnet. Skala: $1=$ trifft gar nicht zu, $6=$ trifft völlig zu (siehe auch Wißhak und Hochholdinger 2018, S. 226), $\propto=$ Cronbachs Alpha, TR=Trainer/innen $(200>N>198)$, TN = Teilnehmende $(253>N>147)$, $\mathrm{PV}=$ Personalverantwortliche $(93>N>91)$. 
Wissensfacetten bestimmt (siehe Tab. 2). Die Ergebnisse liegen überwiegend im guten bis zufriedenstellenden Bereich, Niedrig sind die Reliabilitätskennwerte für Fachwissen, Wissen über Beratung und Coaching sowie für fachdidaktisches Wissen. Dies ist vermutlich durch die geringen Itemzahlen von zwei bis drei Items pro Dimension bedingt.

Es wurde auch untersucht, ob sich die Ergebnisse aus der Perspektive der einzelnen Stakeholder-Gruppen unterscheiden. Die Ergebnisse der MANOVA weisen darauf hin, dass Trainer/innen, Teilnehmende und Personalverantwortliche die Wissensdimensionen unterschiedlich einschätzen (Pillai-Spur $=0,31, F(20,1034)=9,30, p<0,001)$. Um genauer und simultan zu betrachten, ob und auf welchen Dimensionen sich diese drei Gruppen unterscheiden, wurde eine Diskriminanzanalyse durchgeführt, die teststärker ist als zahlreiche Posthoc-Vergleiche, die sich aus drei Gruppen und 10 Wissensdimensionen ergeben. Die Ergebnisse der Diskriminanzanalyse weisen auf zwei zugrundeliegende Diskriminanzfunktionen hin, wobei die erste $94,3 \%$ der Varianz erklärt $\left(R^{2}=0,28\right)$ und die zweite $5,7 \%\left(R^{2}=0,02\right)$. Zusammen unterteilen sie die Gruppen statistisch signifikant $\left(\Lambda=0,70, \chi^{2}(20)=184,54, p<0,001\right)$. Die erste, bedeutsamere Funktion unterscheidet Trainer/innen und Personalverantwortliche auf der einen Seite und Teilnehmende auf der anderen Seite. Sie lässt sich anhand der im folgenden berichteten Korrelationen zwischen den Diskriminanzvariablen, die hier in den Wissensdimensionen bestehen, und der Diskriminanzfunktion und mit Blick auf die deskriptiven Werte (vgl. Tab. 2) dahingehend interpretieren, dass die Teilnehmenden Fachwissen $(r=0,20)$ und Beratungswissen $(r=0,17)$ als bedeutsamer einschätzen als Trainer/innen und Personalverantwortliche. Außerdem bewerten die Teilnehmenden pädagogisch-didaktische Aspekte, also Wissen über Gruppenmanagement $(r=-0,59)$, über individuelle Unterschiede $(r=-0,49)$, methodische Umsetzung $(r=-0,37)$ und Fachdidaktik $(r=-0,31)$ als weniger wichtig als Trainer/innen und Personalverantwortliche. Je nachdem, welche Akteure man also befragt, scheinen sich die Einschätzungen zu relevantem Trainerwissen leicht zu unterscheiden. Möglicherweise sind die Trainer/innen und die Personalverantwortlichen aufgrund ihrer beruflichen Rolle stärker für die pädagogischen, didaktischen und methodischen Aspekte von Trainings sensibilisiert, während für die Teilnehmenden eher das Fachwissen der Trainer/ innen im Vordergrund steht.

Trotz dieser geringen Unterschiede in der Bewertung durch die drei Gruppen lässt sich an dieser Stelle festhalten, dass die 41 Wissensfacetten überwiegend als bedeutsam für die Trainertätigkeit eingeschätzt wurden. Somit kann ProwisT zukünftig im Sinne eines Reflexionsinstrumentes für die Selbst- oder Fremdeinschätzung des professionellen Handlungswissens von Trainer/innen eingesetzt werden. Auf den konkreten praktischen Nutzen des Instruments wird im letzten Abschnitt dieses Artikels ausführlich eingegangen, nachdem zunächst in Abschnitt vier die Limitationen diskutiert werden.

\section{Limitationen und Forschungsdesiderata}

Bezüglich der Validierung des Instruments ProwisT sind einige Aspekte zu bedenken.

Im Hinblick auf die zehn angenommenen Dimensionen von Trainerwissen ist zunächst kritisch anzumerken, dass es sich hierbei nicht um empirisch überprüfte Dimensionen eines Modells handelt. Vielmehr wurden die einzelnen Wissensfacetten zunächst theoriegeleitet systematisiert. Eine empirische Untersuchung der Dimensionalität des Konstrukts Trainerwissen mittels strukturprüfender Verfahren wäre in zukünftigen Schritten möglich und wünschenswert.

Des Weiteren ist bezüglich der empirischen Befragung zur Relevanz der Wissensfacetten einschränkend zu diskutieren, dass die Bewertungen der Befragten in erster Linie deren subjektive Einschätzung widerspiegeln. Da die Wissensfacetten allerdings auf einer literaturbasierten Anforderungsermittlung beruhen, die sowohl den theoretischen als auch empirischen Forschungsstand einbezog, und keine der Facetten als unwichtig eingeschätzt wurde, kann die Erhebung als zusätzliche inhaltliche Validierung der Facetten betrachtet werden. Darüber hinaus sind weitere Validierungsschritte für das Instrument erforderlich, etwa eine Konstruktvalidierung mit Blick auf die Dimensionen des Trainerwissens und eine Kriteriumsvalidierung, etwa durch die Korrelation der Fragebogenergebnisse mit objektiven Wissenstests oder mit Kriterien der Arbeitsleistung von Trainer/innen. Teilweise könnte dafür der Test des generischen pädagogisch-psychologischen Wissens von Lehrpersonen von Marx et al. (2017) genutzt werden, sowie der kürzlich publizierte Kurzfragebogen zur Beurteilung von Trainerkompetenzen von Grohmann et al. (2021). In letzterem werden die Methoden-, Fach-, Sozial-, und Selbstkompetenz von Trainer/innen durch ihre Trainingsteilnehmenden eingeschätzt. Aspekte des professionellen Handlungswissens, die aufgrund des betrieblichen Kontexts speziell für Trainer/innen wichtig sind, wie etwa das Wissen über Determinanten des Trainingstransfers, werden allerdings auch von diesen beiden Instrumenten nicht erfasst.

Bezüglich des für die Selbst- bzw. Fremdeinschätzung modifizierten Instruments ProwisT ist noch einschränkend zu erwähnen, dass solche Einschätzungen nicht objektiv sind und die Ergebnisse daher nur eine erste Orientierung geben, bzw. als Reflexions- und Diskussionsgrundlage für eine individuelle oder organisationale Kompetenzentwicklung dienen können. Auch müssen beim praktischen Einsatz des Instruments die eingeschränkten Reliabilitäten der Dimensionen Fachwissen und Wissen über Coaching und 
Beratung kritisch berücksichtigt werden. Die obigen Analysen beziehen sich zudem auf die Relevanzeinschätzung der Wissensfacetten. Um die Validität und Reliabilität des Instrumentes für die Selbst- und Fremdeinschätzung empirisch zu prüfen, sind weitere Studien notwendig.

\section{Praktischer Nutzen des Instruments}

Das vorgestellte Instrument ermöglicht die Einschätzung des professionellen Handlungswissens von Trainer/innen und umfasst dabei zehn theoretisch fundierte Wissensdimensionen mit insgesamt 41 Wissensfacetten. ProwisT kann in der Praxis auf vielfältige Weise von Trainer/innen, Trainingsanbietern und Personalentwickler/innen eingesetzt werden.

Im Rahmen der Professionalisierung von Trainer/innen kann der Fragebogen zur individuellen Reflexion und Weiterentwicklung verwendet werden, indem Trainer/innen ihr eigenes Wissen einschätzen. Wenn der Fragebogen geringfügig umformuliert wird, kann diese Selbsteinschätzung durch Beurteilungen von Ausbilder/innen, Personalentwickler/innen oder Trainerkolleg/innen ergänzt und mit ihnen abgeglichen und gemeinsam besprochen werden. Dabei setzt die Bewertung durch Dritte voraus, dass diese Personen z. B. durch eine enge Zusammenarbeit oder eine Hospitation in der Lage sind, das Wissen der Trainer/in in den Bereichen einzuschätzen.

Auf der Basis dieser Selbst- und/oder Fremdeinschätzung kann im Sinne einer Bedarfsanalyse ein individueller Weiterbildungsplan entwickelt und zu einem späteren Zeitpunkt erneut evaluiert werden. Somit kann das Instrument auch im Rahmen eines organisationalen Kompetenzmanagements (Kauffeld und Paulsen 2018) verwendet werden, um einen Abgleich zwischen Soll- und Ist-Zustand vorzunehmen. Der Soll-Zustand wäre jeweils die höchste Ausprägung auf der Skala, da die Wissensfacetten aufgrund empirischer Befunde für lern- und transferförderliche Maßnahmen erstellt wurden. Darüber hinaus können Organisationen aber auch selbst ermitteln, ob einzelne Facetten für ihre Trainingsbedarfe mehr oder weniger wichtig sind. Auf Grundlage dieses Soll-Ist-Abgleiches können die nächsten Schritte für die individuelle Weiterentwicklung interner Trainer/innen im Sinne der Unternehmensstrategie abgeleitet werden. Darüber hinaus können Curricula von Train-the-Trainer Angeboten oder Inhalte von betriebsinternen Weiterbildungen für interne Trainer/innen an den einzelnen Wissensdimensionen und -facetten ausgerichtet werden. Befunde von Wißhak et al. (2020) weisen darauf hin, dass ein großer Anteil der Trainer/innen eine Train-theTrainer Ausbildung absolviert. Sie stellt somit einen wichtigen Baustein für die Qualifizierung von Trainer/innen dar. Dabei kann der Fragebogen auch für die Evaluation der
Trainerausbildungen eingesetzt werden, indem er vor und nach der Ausbildung ausgefüllt wird.

Weiterhin kann das Instrument bei der Auswahl und Einstellung von Trainer/innen verwendet werden. Die einzelnen Wissensdimensionen und -facetten können als Grundlage für ein auf den individuellen Bedarf eines Unternehmens angepasstes Anforderungsprofil (Blickle 2019) verwendet werden, das als Basis für die Konzeption von Personalauswahlverfahren genutzt werden kann.

Das Instrument bietet damit breite Einsatzmöglichkeiten, um die Kompetenzentwicklung und damit die Professionalisierung von Trainer/innen auf individueller und organisationaler Ebene zu unterstützen.

Funding Open Access funding enabled and organized by Projekt DEAL.

Open Access Dieser Artikel wird unter der Creative Commons Namensnennung 4.0 International Lizenz veröffentlicht, welche die Nutzung, Vervielfältigung, Bearbeitung, Verbreitung und Wiedergabe in jeglichem Medium und Format erlaubt, sofern Sie den/die ursprünglichen Autor(en) und die Quelle ordnungsgemäß nennen, einen Link zur Creative Commons Lizenz beifügen und angeben, ob Änderungen vorgenommen wurden.

Die in diesem Artikel enthaltenen Bilder und sonstiges Drittmaterial unterliegen ebenfalls der genannten Creative Commons Lizenz, sofern sich aus der Abbildungslegende nichts anderes ergibt. Sofern das betreffende Material nicht unter der genannten Creative Commons Lizenz steht und die betreffende Handlung nicht nach gesetzlichen Vorschriften erlaubt ist, ist für die oben aufgeführten Weiterverwendungen des Materials die Einwilligung des jeweiligen Rechteinhabers einzuholen.

Weitere Details zur Lizenz entnehmen Sie bitte der Lizenzinformation auf http://creativecommons.org/licenses/by/4.0/deed.de.

\section{Literatur}

Arthur Jr, W., Bennett Jr, W., Edens, P. S., \& Bell, S. T. (2003). Effectiveness of training in organizations. A meta-analysis of design and evaluation features. Journal of Applied Psychology, 88, 234-245. https://doi.org/10.1037/0021-9010.88.2.234.

Baldwin, T. T., \& Ford, J. K. (1988). Transfer of training: a review and directions for future research. Personnel Psychology, 41, 63-105. https://doi.org/10.1111/j.1744-6570.1988.tb00632.x.

Baumert, J., \& Kunter, M. (2006). Stichwort: Professionelle Kompetenz von Lehrkräften. Zeitschrift für Erziehungswissenschaft, 9, 469-520.

Bell, B.S., Tannenbaum, S.I., Ford, J.K., Noe, R. A., \& Kraiger, K. (2017). 100 years of training and development research. What we know and where we should go. The Journal of Applied Psychology, 102, 305-323. https://doi.org/10.1037/ap10000142.

Blickle, G. (2019). Anforderungsanalyse. In F. W. Nerdinger, G. Blickle \& N. Schaper (Hrsg.), Arbeits-und Organisationspsychologie (S. 235-249). Berlin, Heidelberg: Springer.

Blömeke, S., Hsieh, F.-J., Kaiser, G., \& Schmidt, W. H. (Hrsg.). (2014). International perspectives on teacher knowledge, beliefs and opportunities to learn. Dordrecht: Springer. https://doi.org/10.1007/ 978-94-007-6437-8.

Blömeke, S., Gustafsson, J.-E., \& Shavelson, R. J. (2015). Beyond dichotomies. Zeitschrift für Psychologie, 223(1), 3-13. https://doi. org/10.1027/2151-2604/a000194. 
Blume, B. D., Ford, J. K., Baldwin, T. T., \& Huang, J. L. (2010). Transfer of training: a meta-analytic review. Journal of Management, 36, 1065-1105. https://doi.org/10.1177/0149206309352880.

Blume, B.D., Ford, K. J., Surface, E. A., \& Olenick, J. (2019). A dynamic model of training transfer. Human Resource Management Review, 29, 270-283. https://doi.org/10.1016/j.hrmr.2017.11.004.

Bonnes, C., Wißhak, S., \& Hochholdinger, S. (2019). Towards a model of professional competence of workplace trainers. In M. Pilz, K. Breuing \& S. Schumann (Hrsg.), Berufsbildung zwischen Tradition und Moderne. Festschrift für Thomas Deißinger zum 60. Geburtstag (S. 141-158). Wiesbaden: Springer. https://doi.org/ 10.1007/978-3-658-24460-6_9.

Bosche, B., Strauch, A., Schneider, M., \& Brandt, P. (2019). GRETAAnerkennungsverfahren: adaptiv statt one-fits all. Kompetenzvalidierung von Lehrenden zwischen diversen Ansprüchen und Nutzenvorstellungen. Magazin erwachsenenbildung.at, 37(2), 061-06-11.

Burke, L. A., \& Hutchins, H. M. (2008). A study of best practices in training transfer and proposed model of transfer. Human Resource Development Quarterly, 19, 107-128. https://doi.org/10.1002/ hrdq. 1230.

Colquitt, J. A., LePine, J. A., \& Noe, R. A. (2000). Toward an integrative theory of training motivation: a meta-analytic path analysis of 20 years of research. The Journal of Applied Psychology, 85, 678-707. https://doi.org/10.1037/0021-9010.85.5.678.

Driskell, J.E., Willis, R.P., \& Copper, C. (1992). Effect of overlearning on retention. The Journal of applied psychology, 77, 615-622. https://doi.org/10.1037/0021-9010.77.5.615.

Ford, J.K., Baldwin, T. T., \& Prasad, J. (2018). Transfer of training. The known and the unknown. Annual Review of Organizational Psychology and Organizational Behavior, 5, 201-225. https://doi. org/10.1146/annurev-orgpsych-032117-104443.

Fuchs, S. (2011). Professionalitätsentwicklung des Weiterbildungspersonals. Tätigkeiten, Kompetenzen und Fortbildung von Trainern in der beruflichen / betrieblichen Weiterbildung. Hamburg: Dr. Kovac.

Gauld, D., \& Miller, P. (2004). The qualifications and competencies held by effective workplace trainers. Journal of European Industrial Training, 28(1), 8-22. https://doi.org/10.1108/ 03090590410513866.

Gegenfurtner, A. (2011). Motivation and transfer in professional training: A meta-analysis of the moderating effects of knowledge type, instruction, and assessment conditions. Educational Research Review, 6, 153-168. https://doi.org/10.1016/j.edurev.2011. 04.001.

Gegenfurtner, A., Veermans, K., \& Vauras, M. (2013). Effects of computer support, collaboration, and time lag on performance selfefficacy and transfer of training: a longitudinal meta-analysis. Educational Research Review, 8, 75-89. https://doi.org/10.1016/ j.edurev.2012.04.001.

Gegenfurtner, A., Könings, K.D., Kosmajac, N., \& Gebhardt, M. (2016). Voluntary or mandatory training participation as a moderator in the relationship between goal orientations and transfer of training. International Journal of Training and Development, 20, 290-301. https://doi.org/10.1111/ijtd.12089.

Grohmann, A., Schulte, E.-M., \& Kauffeld, S. (2021). Entwicklung und Erprobung eines Kurzfragebogens zur Beurteilung von Trainer/innenkompetenzen in beruflichen Weiterbildungsmaßnahmen. Gruppe. Interaktion. Organisation. Zeitschrift für Angewandte Organisationspsychologie (GIO). https://doi.org/10.1007/ s11612-021-00562-0.

Hodge, S., \& Harvey, J. (2015). Learning and development expertise. An Australian analysis. International Journal of Training and Development, 19, 270-286. https://doi.org/10.1111/ijtd.12062.

Huang, J. L., Blume, B. D., Ford, J. K., \& Baldwin, T. T. (2015). A tale of two transfers. Disentangling maximum and typical transfer and their respective predictors. Journal of Business and Psychology, 30, 709-732. https://doi.org/10.1007/s10869-014-9394-1.

Hughes, A.M., Zajac, S., Woods, A.L., \& Salas, E. (2020). The role of work environment in training sustainment: a meta-analysis. Human Factors, 62, 166-183. https://doi.org/10.1177/ 0018720819845988.

Hunter, J.E. (1986). Cognitive ability, cognitive aptitudes, job knowledge, and job performance. Journal of Vocational Behavior, 29(3), 340-362. https://doi.org/10.1016/0001-8791(86)90013-8.

IBM Corp (2020). IBM SPSS statistics for Windows (version 27.0) [computer software. Armonk: IBM Corp. www.ibm.com.

Kauffeld, S., \& Paulsen, H. (2018). Kompetenzmanagement in Unternehmen: Kompetenzen beschreiben, messen, entwickeln und nutzen. Stuttgart: Kohlhammer.

Keith, N., \& Frese, M. (2008). Effectiveness of error management training. A meta-analysis. The Journal of Applied Psychology, 93, 59-69. https://doi.org/10.1037/0021-9010.93.1.59.

König, J., \& Blömeke, S. (2009). Pädagogisches Wissen von angehenden Lehrkräften. Zeitschrift für Erziehungswissenschaft, 12(3), 499-527. https://doi.org/10.1007/s11618-009-0085-z.

Kunina-Habenicht, O., Lohse-Bossenz, H., Kunter, M., Dicke, T., Förster, D., Gößling, J., Schulze-Stocker, F., Schmeck, A., Baumert, J., Leutner, D., \& Terhart, E. (2012). Welche bildungswissenschaftlichen Inhalte sind wichtig in der Lehrerbildung? Zeitschrift für Erziehungswissenschaft, 15(4), 649-682. https://doi.org/10.1007/ s11618-012-0324-6.

Kunter, M., Baumert, J., Blum, W., Klusmann, U., Krauss, S., \& Neubrand, M. (Hrsg.). (2013). Cognitive activation in the mathematics classroom and professional competence of teachers. Results from the COACTIV project. New York, Heidelberg: Dordrecht, London: Springer.

Lencer, S., \& Strauch, A. (2016). Das GRETA-Kompetenzmodell für Lehrende in der Erwachsenen- und Weiterbildung. https://www. die-bonn.de/doks/2016-erwachsenenbildung-02.pdf. Zugegriffen: 1. Juni 2020.

Marx, C., Goeze, A., Voss, T., Hoehne, V., Klotz, V. K., \& Schrader, J. (2017). Pädagogisch-psychologisches Wissen von Lehrkräften aus Schule und Erwachsenenbildung. Entwicklung und Erprobung eines Testinstruments. Zeitschrift für Erziehungswissenschaft, 20(Suppl 1), 165-200. https://doi.org/10.1007/s11618017-0733-7.

Matsuo, M. (2014). Instructional skills for on-the-job training and experiential learning. An empirical study of Japanese firms. International Journal of Training and Development, 18, 225-240. https:// doi.org/10.1111/ijtd.12035.

Mayring, P. (2010). Qualitative Inhaltsanalyse: Grundlagen und Techniken (11. Aufl.). : Beltz.

Nisar, P. J., \& Scott, H. J. (2011). Key attributes of a modern surgical trainer: perspectives from consultants and trainees in the United Kingdom. Journal of Surgical Education, 68, 202-208. https:// doi.org/10.1016/j.jsurg.2010.12.010.

Reinhold, S., Gegenfurtner, A., \& Lewalter, D. (2018). Social support and motivation to transfer as predictors of training transfer: testing full and partial mediation using meta-analytic structural equation modelling. International Journal of Training and Development, 22, 1-14. https://doi.org/10.1016/j.jsurg.2010.12.010.

Taylor, P. J., Russ-Eft, D. F., \& Chan, D. W. L. (2005). A Meta-analytic review of behavior modeling training. Journal of Applied Psychology, 90, 692-709. https://doi.org/10.1037/0021-9010.90.4.692.

Voss, T., Kunter, M., Seiz, J., Hoehne, V., \& Baumert, J. (2014). Die Bedeutung des pädagogisch-psychologischen Wissens von angehenden Lehrkräften für die Unterrichtsqualität. Zeitschrift für Pädagogik, 60, 184-201.

Weinert, F.E. (Hrsg.). (2002). Leistungsmessungen in Schulen. Weinheim: Beltz.

Wißhak, S., \& Hochholdinger, S. (2018). Trainers' knowledge and skills from the perspective of trainers, trainees and human resource development practitioners. International Journal of Training 
Research, 16, 218-231. https://doi.org/10.1080/14480220.2018. 1576327.

Wißhak, S., Bonnes, C., Keller, I., Barth, D., \& Hochholdinger, S. (2020). Qualifikationen von Lehrenden in der beruflich-betrieblichen Weiterbildung. Zeitschrift für Bildungsforschung, 10, 103-123. https://doi.org/10.1007/s35834-020-00262-7.

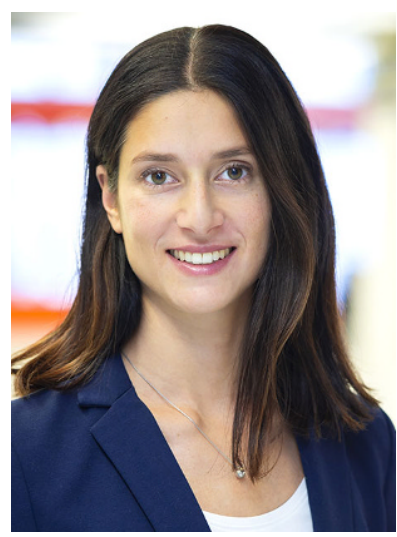

Dr. Susanne Wißhak ist DiplomPädagogin und wissenschaftliche Mitarbeiterin am Lehrstuhl für Betriebspädagogik der Universität Konstanz. Ihre Forschungsschwerpunkte liegen auf Erwachsenenbildung, berufsbezogener Weiterbildung und der Professionalisierung des Weiterbildungspersonals. Nationale und internationale Publikationen weisen sie als Expertin für berufliche und betriebliche LehrLern-Prozesse aus.

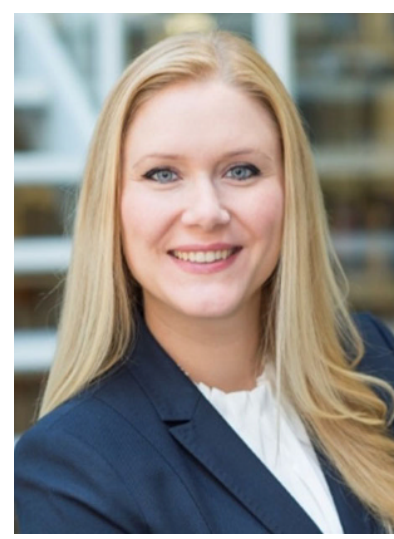

Dr. Caroline Bonnes ist DiplomPädagogin und wissenschaftliche Mitarbeiterin am Lehrstuhl für Betriebspädagogik an der Universität Konstanz. Ihr Arbeitsschwerpunkt ist die empirische Weiterbildungsforschung, mit speziellem Fokus auf der professionellen Handlungskompetenz von Lehrenden, der Gestaltung von Lehr-Lern-Prozessen in der der Weiterbildung und damit zusammenhängend die Förderung des Weiterbildungstransfers.

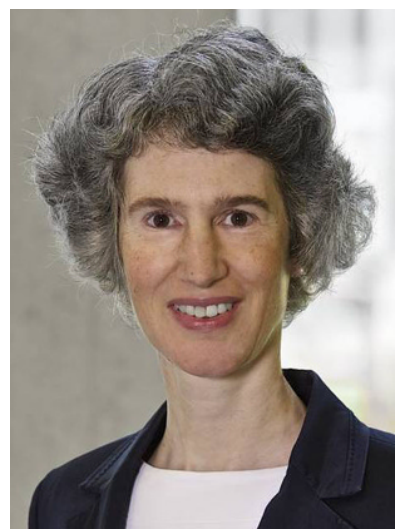

Sabine Hochholdinger ist $\mathrm{Di}$ plom-Psychologin und Inhaberin des Lehrstuhls für Betriebspädagogik an der Universität Konstanz. Sie erforscht die Gestaltung und Evaluation betrieblicher Personalentwicklung und -auswahl, Kompetenzen betrieblichen Weiterbildungspersonals und Lernen mit neuen Medien. Praktische Erfahrungen hat sie mit Trainings und Personalauswahl in Unternehmen erworben. 